Mean Places of the Comparison Stars for the Beginning of the respective Vears of Observations.

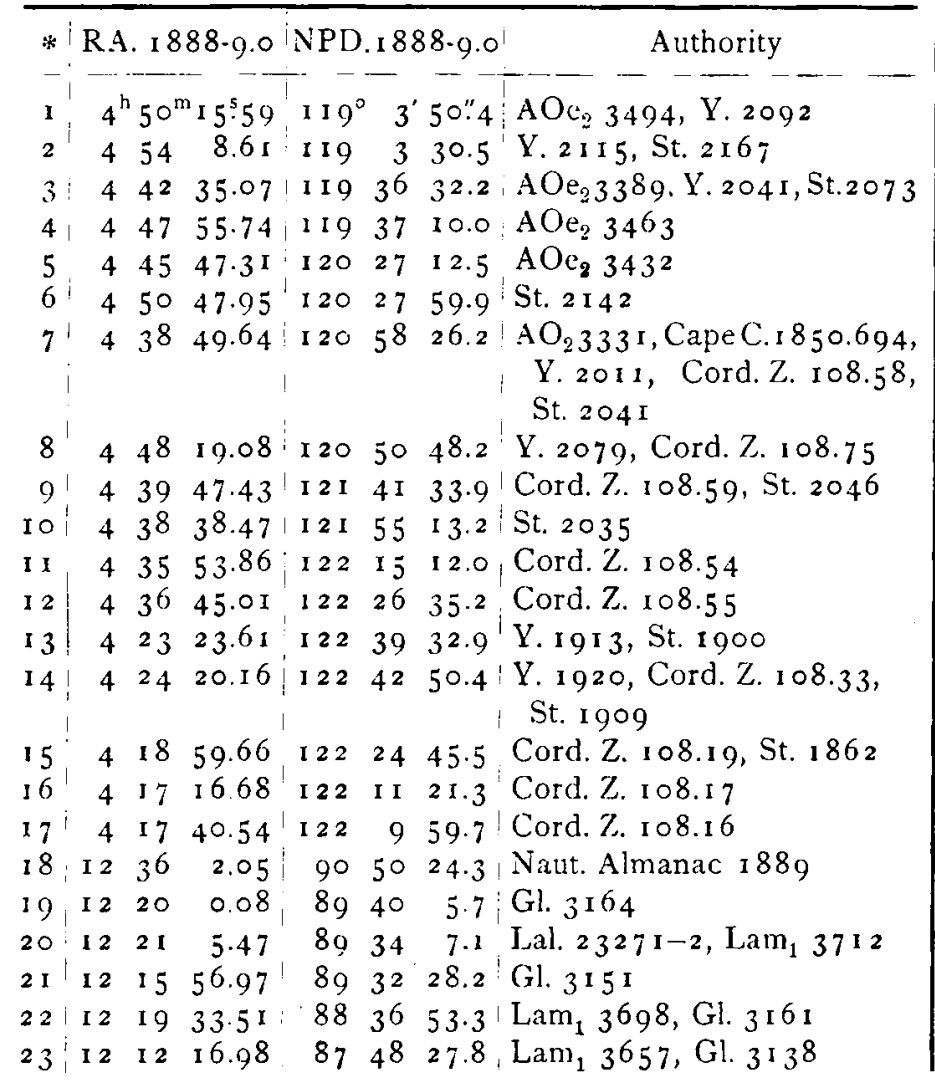

Private Observatory, Windsor N. S. Wales, I89o January I 6.

\begin{tabular}{|c|c|c|c|c|c|c|c|}
\hline$\because$ & \multicolumn{3}{|c|}{ RA. 1889.0} & \multicolumn{4}{|c|}{ NPD. $1889.0^{\prime} \quad$ Authority } \\
\hline 24 & 28 & & ${ }^{m} 26.90$ & $87^{\circ}$ & ${ }^{\circ} 40$ & I 5.6 & Anonymous. Equat. Comps. \\
\hline 25 & I 2 & 8 & 15.83 & 87 & 7 & I 8.1 & Sj. 44 I $2-3$, Gl. 3 I 28 \\
\hline 26 & 12 & 5 & $59 \cdot 57$ & 85 & I 9 & $3^{6.4}$ & $\begin{array}{l}\text { Y. } 5086, \text { Sj. } 4401-2,9 y r . \\
\text { I } 117, \text { Gl. } 3117\end{array}$ \\
\hline 27 & 22 & 5 & 13.14 & 109 & 28 & 13.7 & Cinc. 3725 \\
\hline 28 & 22 & 3 & 14.02 & 109 & 31 & 49.8 & Cinc. 3718 \\
\hline $29 !$ & 22 & $\mathbf{I}$ & 18.32 & IIO & 6 & $\begin{array}{r}33.8 \\
\quad !\end{array}$ & $\begin{array}{l}\mathrm{AOe}_{2} 2 \mathrm{r} 896, \mathrm{Y} .9684 \text {, Cinc. } \\
37 \text { I }\end{array}$ \\
\hline 30 & 22 & 7 & 22.02 & 110 & 7 & 27.4 & Cinc. 3733 \\
\hline $3 \mathbf{I}$ & 22 & 4 & 40.89 & I 10 & 42 & 19.4 & Cinc. 3724 \\
\hline 32 & 21 & $5^{8}$ & 16.95 & I IO & 56 & 56.1 & $\mathrm{AOc}_{2} 21850$, Cinc. 3704 \\
\hline 33 & 22 & $\mathbf{I}$ & $29 \cdot 5^{6}$ & I I I & 18 & $1.2 \vdots$ & Cinc. 3712 \\
\hline 34 & 21 & 28 & $3^{6.99}$ & 110 & 34 & $\begin{array}{r}42 \cdot 5 \\
;\end{array}$ & $\begin{array}{l}\text { Cape C. I } 850.43 \text { I 3, Y. } 94 \text { I } 4 \text {, } \\
9 \text { yr. } 20 \text { I } 4, \text { Rad.Obs. } 1885 . \\
\text { 1 } 882\end{array}$ \\
\hline 35 & 21 & 28 & 40.53 & 110 & 44 & $34.9 !$ & Cape Cat. 1850.4314 \\
\hline $3^{6}$ & $2 \mathrm{I}$ & 28 & 36.97 & I IO & 56 & I 5.1 & Cinc. 3629 \\
\hline 37 & $2 \mathrm{I}$ & 12 & 47.10 & 111 & 45 & 21.4 & $\mathrm{AOe}_{2} 2$ I 293 , Cinc. $35^{82}$ \\
\hline $3^{8}$ & $2 \mathrm{I}$ & 20 & $57 \cdot 30$ & I I I & 40 & $3 I .6$ & $\begin{array}{l}\text { Cape Cat. I } 850.4290, \mathrm{~N} 7 \mathrm{yr} . \\
243 \mathrm{I}, 9 y \mathrm{yr} 2003\end{array}$ \\
\hline 39 & 2 I & 12 & 45.68 & I I I & 44 & 39.9 & $\mathrm{AOe}_{2} 21292$, Cinc. $35^{81}$ \\
\hline 40 & 2 I & I 5 & 36.74 & I I 2 & 0 & I 5. I & Cinc. 3595 \\
\hline $4 \mathrm{I}$ & 2 I & I 9 & 22.43 & 112 & 8 & 26.2 & $\mathrm{AOe}_{2} 2{ }^{2} 37^{2}$, Cinc. $3^{60} 3$ \\
\hline 42 & $2 \mathrm{I}$ & 8 & 41.44 & I I 2 & 16 & 27.2 & Cape Cat. 1850.4236 \\
\hline & & IO & 55.57 & & I 2 & 12.0 & 3570 \\
\hline
\end{tabular}

Fohn Tebbutt.

\title{
Observations of (80) Sappho and Comparison Stars
} during the opposition of 1889 on the Meridian Circle of the Cincinnati Observatory.

(8o) Sappho.

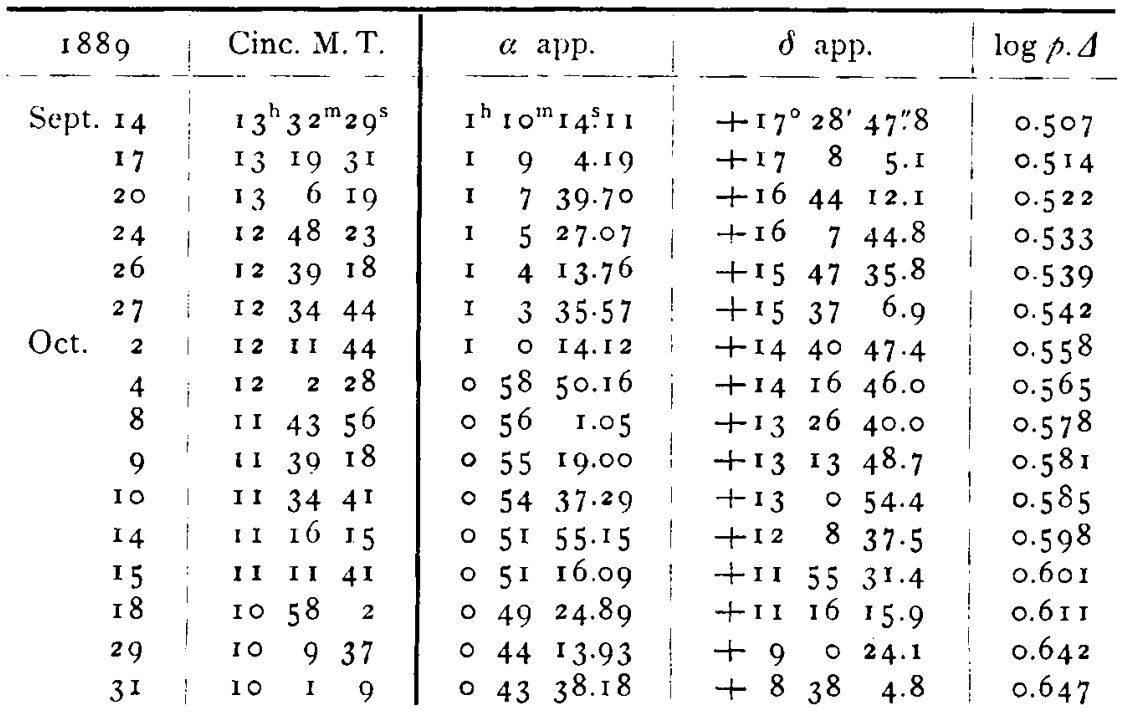


Comparison Stars.

\begin{tabular}{|c|c|c|c|c|c|c|c|c|}
\hline DM. & Aag & & 880.0 & \multicolumn{3}{|c|}{$\delta 1889$} & & \\
\hline$-10: 65$ & $7 \cdot 3$ & $\mathrm{O}^{\mathrm{h}} 3^{\mathrm{r}}$ & ${ }^{n} 32^{s} .04$ & $+10^{\circ}$ & $49^{\prime}$ & $3: 9$ & & \\
\hline 15.106 & $7 \cdot 3$ & 37 & 4.29 & 16 & 3 & 3 & & \\
\hline 0 & 7.7 & 42 & 25.20 & 8 & 36 & 57.1 & & \\
\hline 9.90 & 9.0 & 42 & 31.03 & 9 & 39 & & & \\
\hline 11.102 & 8.6 & 44 & $24 \cdot 3^{6}$ & 11 & 13 & $34 \cdot 7$ & & \\
\hline 9.97 & 8.5 & 44 & 46.74 & 9 & $4 \delta$ & 24.4 & & \\
\hline 11.106 & 7.0 & 45 & 46.17 & I 2 & 10 & 51.8 & & \\
\hline 9.99 & $8 \cdot 3$ & 46 & $20.3^{6}$ & 9 & 59 & 52.0 & & \\
\hline 12.104 & $7 \cdot 3$ & 46 & 40.01 & 13 & 2 & 42.8 & & \\
\hline 9.101 & 8.3 & 47 & $0.5^{6}$ & 9 & 12 & 4.6 & & \\
\hline 10.105 & 8.4 & 48 & 2.89 & 10 & 31 & 12.5 & & \\
\hline 12.108 & 8.2 & 4.9 & II.93 & 12 & 14 & 44.7 & & \\
\hline 13.127 & 6.5 & 5 & 19.66 & I 3 & 21 & 1.0 & & \\
\hline I I. I 18 & 8.8 & 50. & $.40 .3^{8}$ & 12 & 6 & 43.6 & & \\
\hline 13.130 & 8.7 & 50 & 52 & 14 & IO & $29 \cdot 5$ & & \\
\hline 9.110 & 8.7 & $5^{\circ}$ & 59 & 9 & 24 & 53.8 & & \\
\hline I 1.120 & 8.2 & 51 & 32.90 & 11 & 49 & $4^{6.2}$ & & \\
\hline 12.119 & 6.0 & 52 & 4 & 13 & 5 & 4 & & \\
\hline .143 & 8.5 & & 53 & 14 & 0 & $3^{8.0}$ & & \\
\hline .115 & 8.2 & 55 & 25.67 & 10 & 34 & 58.8 & & \\
\hline J & 8.0 & & $3^{2.84}$ & 11 & 18 & 51.6 & & \\
\hline 13.150 & 8.5 & 056 & $43 \cdot 5^{2}$ & +13 & 39 & $17 \cdot 3$ & & \\
\hline
\end{tabular}

\begin{tabular}{|c|c|c|c|c|c|c|c|c|c|}
\hline $\mathrm{DM}$ & Mag. & \multicolumn{3}{|c|}{$\alpha \quad 1889.0$} & \multicolumn{3}{|c|}{$\delta$ I 889.0} & \multicolumn{2}{|c|}{ Obs } \\
\hline$-15^{\circ} \mathrm{I} 54$ & 8.5 & 0 & $57^{\pi}$ & 9.64 & +15 & $3^{2}$ & $27: 2$ & & 3 \\
\hline I2.1 26 & 7.8 & & 57 & I 6.36 & 12 & 27 & 9.8 & & 4 \\
\hline 13.155 & 8.7 & & $5^{8}$ & 0.52 & 13 & 20 & 26.2 & & 3 \\
\hline 14.163 & 5.5 & & 59 & 13.72 & 14 & 20 & 55.9 & & 7 \\
\hline 14.168 & 9.0 & o & 59 & 57.05 & 14 & 23 & 14.6 & & 5 \\
\hline 14.169 & 7.7 & I & $\circ$ & $4 \cdot 3^{6}$ & 14 & 47 & 23.6 & & 4 \\
\hline 15.159 & 8.5 & & o & 29.60 & I 5 & 43 & 9.8 & & 3 \\
\hline 15.164 & 8.0 & & I & $59 \cdot 56$ & I 5 & I 6 & 15.1 & & 5 \\
\hline I 6.1 .16 & 8.0 & & 2 & 2.07 & 16 & 49 & II. 3 & & 3 \\
\hline I 6.1 I 9 & 8.6 & & 2 & 34.82 & I 7 & 3 & 40.3 & & 4 \\
\hline I 4.175 & 6.4 & & 4 & 18.29 & I 5 & 4 & $5^{8.2}$ & & 3 \\
\hline 17.166 & 8.8 & & 5 & 3.82 & 17 & 48 & 6.6 & & 4 \\
\hline I 6.123 & 8.5 & & 5 & 10.19 & 16 & I I & 12.4 & & 5 \\
\hline 13.175 & 8.2 & & 5 & 23.95 & 14 & 6 & 4.9 & & 4 \\
\hline 15.175 & 8.5 & & 6 & $55.7 \mathrm{I}$ & 16 & 10 & 29.3 & & 3 \\
\hline 15.177 & 6.5 & & 8 & 13.81 & 15 & 32 & 44.5 & & 3 \\
\hline I 6.129 & 83 & & Io & 15.80 & 17 & 2 & 53.6 & & 4 \\
\hline 15.185 & 8.8 & & I I & 34.26 & 15 & 46 & $7 \cdot 4$ & & 4 \\
\hline 17.183 & 8.0 & & 12 & 26.02 & 17 & 59 & $31 \cdot 3$ & & 4 \\
\hline $10.17 \mathrm{I}$ & 7.0 & & 17 & 2. I 5 & 10 & 47 & 13.5 & & 21 \\
\hline+16.154 & 7.2 & I & 22 & 25.96 & +16 & 30 & I 6.5 & & 22 \\
\hline
\end{tabular}

These positions depend on the stars of the Berliner Jahrbuch. Each star was observed with circle both east and west. The average probable error of one observation is \pm 0.050 and \pm 0.44 respectively.

\section{G. Porter, Director.}

\section{Elliptische Elemente des Cometen 1889 IV und Bemerkungen über einige andere Cometen.}

\section{Von A. Berberich.}

Von dem Cometen i 889 IV hat Mr. Campbell im Astr. J. IX.1 19 ein elliptisches Elementensystem mit einer Umlaufszeit von etwa 3000 Jahren mitgetheilt, das aus drei Beobachtungen 1889 Juli 23, Aug. I 9 und Sept. 27 abgeleitet ist. Dieses Resultat wird durch die folgende Rech. nung im Wesentlichen bestätigt; es war nicht möglich, durch eine Parabel den beobachteten Lauf des Cometen befriedigend darzustellen. Meine Elemente beruhen auf sechs Oertern: I. Juli 23 , Melbourne; II. Juli 3 I, Wien, Dresden, München, Mailand, Kiel ; III. Aug. 30, Dresden, Algier; IV. Sept. 24 , München, Padua; V. Oct. I6, München; VI. Nov. 21 , Wien. Auf das mittl. Aequ. 1889.0 bezogen, lauten diese Positionen :

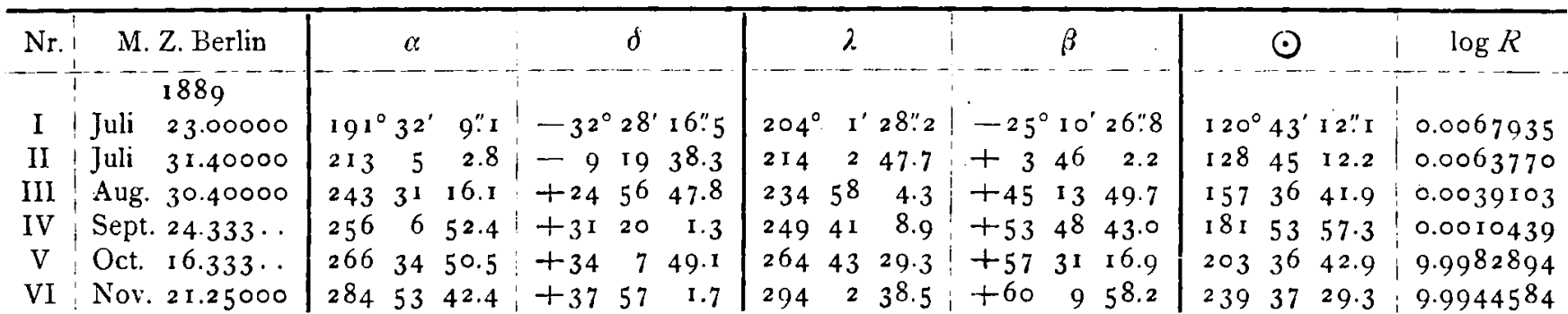
Elementensystem :

Durch Variation der zu Juli 3 I und Nov. 2 I gehörenden geocentrischen Distanzen erlangte ich folgendes

$$
\begin{aligned}
& T=\text { 1889 Juli 19.3108 I M. Z. Berlin } \\
& \omega=345^{\circ} 5^{1^{\prime}} \cdot 57^{\prime \prime} 6 \\
& \Omega=286 \quad 947.0 \quad \text { M. Aequ. } 1889.0 \\
& i=655^{8} 4^{\text {I.I }}
\end{aligned}
$$$$
\begin{aligned}
\log q & =0.016890 \\
\log e & =9.998479 \\
\log a & =2.47325 \\
U & =5 \text { 127 Jahre }
\end{aligned}
$$ 